\title{
The Constraint-based Computer Editing Techniques of the Athlete Action Image
}

\author{
Guo Sheng \\ P.E.College, Beihua University \\ Jilin City, Jilin Province, China
}

Keywords: constraint ; athlete action ; editing techniques motion editing ; key frame

\begin{abstract}
With the development of the motion capture technology, abundant realistic human motion capture data have been widely used in many areas of the computer screen making. Tracking, capturing and analyzing human motion, we can further study each link of the athlete action techniques to help the quantitative analysis. Meanwhile, combined with human physiology and sports biomechanics, the training methods can be improved. Therefore, the movement training doesn't depend on experience, instead, comes into a theorized and digital age. The constraint-based editing techniques, which alter motions, preserve specific spatial features. This paper researches the basic theory of human motion editing and describes the motion techniques of the human hierarchical joint model. On the base of researching the key frame editing and processing method, a small motion editing system can be formed by using Matlab tool to achieve various technical simulations.
\end{abstract}

\section{Introduction}

With computers increasingly widely used in all of life, the computer animation comes into sports field. Although motion capture technology can capture realistic human motion date, yet various motion types, complex body structure and many other reasons request us to deal with the capture data. Then we can find useful date, which makes motion editing techniques become an important research subject, The main feature of the constraint-based motion editing algorithm is that, in the editorial process, some characteristics of the motion data and the requirement of the customers are constrained and preserved. The constraint- based editing methods are combined with the inverse kinematics and the users set different constraints to generate new motions. The users don't have to be too much involved in the whole process. This kind of operation is mainly used in motion transformation. In making motion pictures, the constraint-based motion editing has a broad prospect of application and great practical value of improving athlete performance and providing scientific training data.

\section{The description of human movement model}

Before editing the athlete action, it is necessary to establish an appropriate body model. If the body model is too simple, some important movement detail information will be lost; it is too complex, it will increase the application and the solving difficulty. There is a common skeleton model used in the computer animation of the human hierarchical skeleton model, which is formed on the basis of the analysis of baby skeleton structure and makes it simply simulated. Using this model not only helps to control the action, but also reflects the characteristics of sports information. The body skeleton structure is described by the hierarchical skeleton model, which can show the corresponding tree structure and the number of each joint freedom. Under this condition, the human body posture can be expressed as a translation and rotation vector. The body location is controlled by the root node balance and the body action is controlled by the rotation of each joint node. The 3d vector is used to express the translation. Euler angle, the rotation matrix and quaternion are used to 
express the rotation. The body movement can be expresses as the function of body posture changing over time. That is:

$M(t)=(\operatorname{Troot}(t), \operatorname{Rroot}(t), R 1(t), A, R n(t)$

Among them, Troot $(\mathrm{t})$ and $\operatorname{Root}(\mathrm{t})$ mean translation and rotation of the root joints respectively, $\mathrm{Ri}(\mathrm{t})$ means i joint rotation. According to the translation and rotation, each joint position can be worked out. The solution formulas of the left foot joint position are as follows:

Plfoot(x1, y2, z3) =Troot Rroot Tlfemur Rlfemur Tltibia Rltibia Tlfoot Plfoot(x0, y0, z0)

Among them, Plfoot(x1, y2, z3) means the left foot position at moment in the world coordinate. Plfoot(x0, y0, z0) means the local coordinate of the left foot joint with its parent joint for origin in the local coordinate system at the initial time. Ti expresses the parallel vector of the current coordinate system translated to another coordinate system with the parent joint for origin.

\section{The constraint-based motion editing techniques}

The constraint-based motion editing techniques describe the motion features required preserving and altering as different detailed constraints. According to the techniques, the athletic performance characteristics can be clearly expressed. For example, if a motion can be expressed as an equation, which specifies the position of the end effector in the given time, this feature is regarded as constraint conditions to keep motion characteristics or to control the motion transformation. The method of time and space constraints thinks about a motion segment in a calculation. The solver of this method calculates the whole segmental motion at a time, or a word motion of it. So this method considers not only constraint conditions, but also the target standard of the whole segmental motion. Time and space constraints were originally used to specify the expected position of the role, and then with the help of the solver, can make these postures in the best motion. Time and space constraints also meet the constraints of the physical laws, create a target function and define the best solution of the minimal energy cost to meet the role. The motion worked out by this method can meet physical correctness. Time and space constraints can be expressed as:

Minimize $g(x)$ subject to $f(x)=c$

Among them, $\mathrm{g}(\mathrm{x})$ and $\mathrm{f}(\mathrm{x})=\mathrm{c}$ are target function and constraints means the motion of minimizing the target function subject to the constraint conditions.

\section{The motion editing method based on key frame}

The first step is to choose the original movement sequence with different features in order to extract the key frame. Following that we can compare the different effects after extracting different threshold. In editing process, the human body is described by the hierarchical skeleton model, the motion postures by translation and rotation, and joint rotation drives skeleton transformation. Following that, take different weight array and compare different extracting results. After finishing key frame extraction, it is edited subject to geometric constraints on the base of the inverse kinematics. Next, by using the interpolation method of quadratic curves, the root node can be translated and interpolated. And then, Slerp interpolation method is used to interpolate joint rotation. Finally, after movement is reconstructed, we should observe its sequence changes to keep its characteristics.

The motion capture data under ASF/AMC format are original data from the motion capture database of Carnegie Mellon University. In the experiment, Matlab is a tool of processing simulation data, Maya software from Turk company is not only used to edit motions, but also a kind of demo tool. The experiment process is as shown in figure 1.

\section{The motion editing system based on Matlab}

The Matlab editing system realized the editing technology based on key frame. This system can input ASF/AMC format data. Through this system, the key frame can be extracted, the movement 
interpolation can be reconstructed, and the movement can be processed according to filter combination and frame algorithm.

Matlab 7.0 is the system development platform, which processes motion data and designs the user interface to make it convenient for the user to edit motions. This system consists of three function modules, one is an editing module based on the key frame, another is a motion module of filter combination and frame algorithm, the third is a data processing module which is used to convert ASF/AMC data to/from Matlab matrix data. The system construction is as shown in figure 2.

The whole work flow of the system is divided into the following steps:

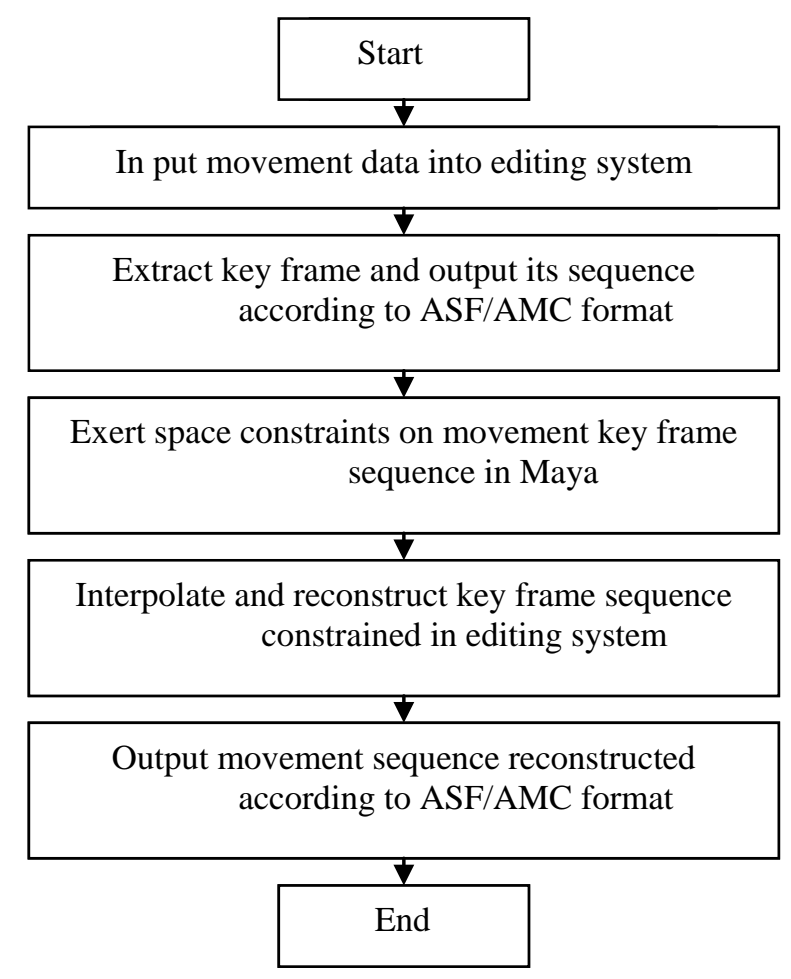

Figure 1. Key frame editing experiment simulation -based flow chart

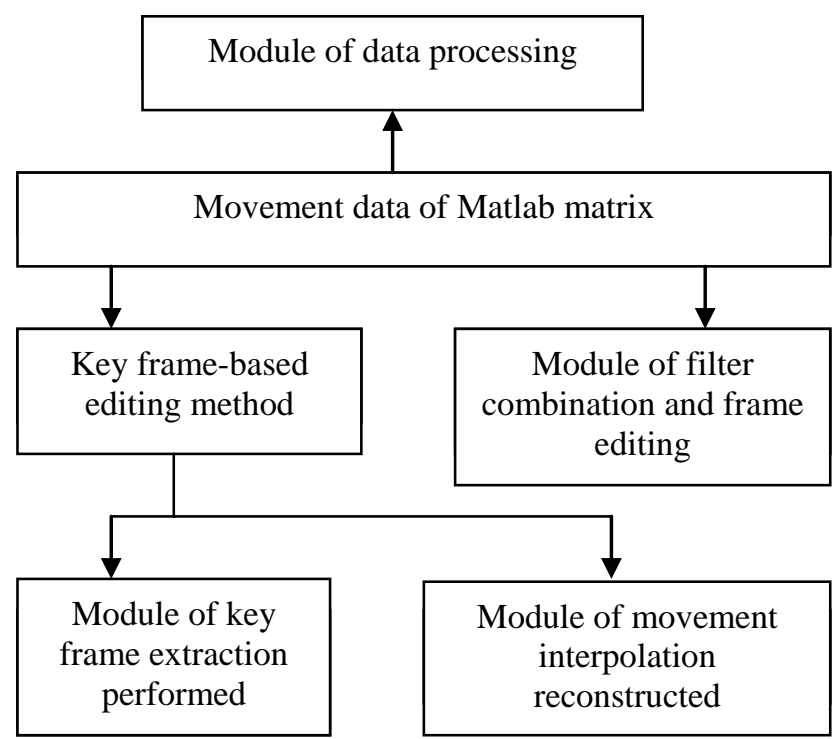

Figure 2. System infrastructure diagram

The first step is input a movement sequence according to the differently selected editing methods. And then, the input movement sequence is conversed its format to be expressed as Matlab matrix 
and processed. Finally, if selecting the method of filter combination and frame editing, we can directly process the movement sequence which will be output in ASF/AMC format.

\section{Summary}

The constraint-based editing techniques, which alter motions, preserve specific spatial features. This paper researches the basic theory of human motion editing and describes the motion techniques of the human hierarchical joint model. So we can achieve better editing effect and provide scientific basis for studying and analyzing the athlete technical movement.

\section{References}

[1] Fischler M A, Bolles R C. Random Sample Consensus: A Paradigm for Model Fitting with Application to Image Analysis and Automated Cartography [J].

[2] Or S H, LuK W S, Wong K H, King I, An Efficient Iterative Pose Estimation Algorithm [Z].

[3] YangWenKai. Sequence image moving targets detection. Northwestern polytechnical university news. 2004

[4] YangHongFeng. Constraint sequence moving objects in real-time division and tracking of the journal of northwestern university. 2003

[5] WangHuaJun. Constraint of image motion of the analysis and extraction, says. Jilin university press. 2004

[6] JinMin, xu punctual. The same moment in the application of pattern recognition. The computer engineering and application. 2004

[7] WangBin. Constraint sequence of target motion detection and tracking the research problem. Northwest university press. 2004

[8] Chen Jiashi, “3 d reconstruction movement under the perspective projection”, computer aided design and graphics journals, 1999.

[9] Wang Rencheng, Huang Changhua, Wang Jijun, Jin Dewen, "Image processing based on the index point of the camera in the human motion analysis system”, tsinghua university press, 1999.

[10] Li Yang "the sport balance function evaluation research progress". Shenyang Sorts institude jurnal. 